\title{
Motivación y actitudes del alumnado universitario al inicio de la carrera. ¿Varían al egresar?
}

\section{Ignacio González López}

Departamento de Educación, Universidad de Córdoba

\section{España}

ed1goloi@uco.es 


\section{Resumen}

Introducción. La finalidad del trabajo aquí presentado se centra en definir cuáles son los factores que afectan a la decisión tomada por un sujeto a la hora de decidirse por cursar unos estudios univesitarios, así como advertir cuáles son los patrones actitudinales que guían su permanencia en la institución.

Método. Centrados en el estudio de los motivos de elección de carrera y las actitudes hacia la Universidad (creencias sobre el contexto y concepción del papel del estudiante) hemos seleccionado dos grupos muestrales (alumnado de nuevo ingreso y a punto de egresar) a los que hemos aplicado una encuesta formada por 32 ítems de valoración escalar, llevando a cabo los diferentes análisis estadísticos clasificando al alumnado en función de variables como el género y la rama de especialización a la que pertenecen sus estudios.

Resultados. Tras la aplicación de diferentes técnicas de análisis de datos (descriptivas e inferenciales) hemos constatado los motivos que guían al alumnado en su proceso de elección de estudios universitarios, destacando la existencia prioritaria de preferencias personales y profesionales. Por otro lado, hay que señalar que no existen evidencias estadísticamente significativas en cuanto a la evolución de las actitudes de los estudiantes hacia la Universidad, reduciendo las mismas al contexto y objetivos de la institución.

Discusión. La puesta en marcha de este estudio nos ha permitido constatar la existencia de un conjunto de motivaciones y actitudes de los que depende tanto la elección de carrera como la calidad de vida de los estudiantes durante el tiempo que duran sus estudios. De este modo, y dando al alumnado la oportunidad de participar de su vida académica, podremos corregir posibles deficiencias en los procesos de orientación y tutoría en los niveles de educación secundaria y educación universitaria.

Palabras-clave: motivación, actitudes, evaluación educativa, educación superior. 


\section{Introducción}

¿Qué es lo que provoca que un individuo decida realizar estudios universitarios? ¿Qué factores están influyendo de forma decisiva en el alumno durante su estancia en la institución universitaria y le motivan para continuar? Cofer y Appley (1993, p. 17) responden que "existe un determinante ambiental que precipita la conducta en cuestión; la urgencia, apetencia, sentimiento, emoción, pulsión, instinto, carencia, deseo, demanda, propósito, interés, aspiración, necesidad, plan o motivo; o el incentivo o meta que atrae o repele al organismo".

En el ámbito empírico, son diferentes los estudios que, en los últimos años, tratan de dar respuesta a estos interrogantes, entre los que destacamos, por un parte, el trabajo realizado por Valle et al. (1999), quienes han propuesto un modelo causal sobre los determinantes del rendimiento académico caracterizado por factores fundamentales como el autoconcepto, las atribuciones y las metas de logro. Por su parte, un trabajo más reciente elaborado por Gámez y Marrero (2003), trata de explorar las metas y motivos de los estudiantes que inician sus estudios en la Universidad, concretándose en factores de tipo afectivo como la afiliciación y los intereses personales, de poder (prestigio) y de logro.

Sin embargo, aceptamos como respuestas válidas para estas cuestiones las aportadas por Mora Ruiz (1990), González (1999), Bricall (2000) y ANECA (2004) que destacan cuáles son los elementos que condicionan y motivan la demanda de este tipo de estudios por parte del alumnado:

1. En primer lugar, la existencia de una serie de factores económicos, importantes para explicar la participación en la educación superior, ya que afectan a la decisión individual de demandarla y afectan a la decisión de la sociedad de sustentar la oferta educativa en unos determinados niveles. El individuo que desea matricularse en un centro superior decide aceptar los costes (directos del estudio: tasas, libros, etc; de mantenimiento en el sentido que se vea obligado a residir fuera del entorno familiar y de oportunidad en el caso de tener que renunciar a un trabajo lucrativo por decidir realizar estudios superiores) si se cumplen dos requisitos: que los beneficios esperados sean superiores a los costes y que su situación económica personal (generalmente la familiar) le permita asumirlos. Sin embargo, la valoración de los beneficios es compleja y personal, ya que puede tratarse de beneficios monetarios futuros, el prestigio que proporcionan los estudios, así 
como el conjunto de beneficios no monetarios que cabe esperar de la adquisición de la educación.

2. Por otro lado, existen un conjunto de factores demográficos como el tamaño y la estructura de las cohortes de edad que se corresponden con los años usuales de los estudios superiores. El número de individuos en edad universitaria ha sido un factor de relevante importancia en estos últimos treinta años. Del mismo modo, la estructura es determinante en el sentido de que elementos como los niveles de educación previa, el género, el lugar de procedencia, etc. no sólo actúan sobre la demanda sino en la respuesta del individuo frente a los cambios producidos en otras variables intervinientes.

3. En tercer lugar, destacan los factores familiares, entre los que se señalan el nivel de educación de los padres, el tipo de profesión de los mismos y el número de hermanos. Parece evidente que el nivel educativo y ocupacional de los padres, a través de la transmisión de valores a los hijos y de la existencia de un mayor o menor ambiente cultural, pueden ejercer un importante estímulo sobre la demanda.

4. Al mismo tiempo, otorgan importancia a los factores sociales, entre los que destacan la creciente diversidad cultural condicionada por los movimientos migratorios y la aceleración de la internacionalización de las sociedades modernas.

5. Igualmente, destacan los factores personales resumidos en tres: la capacidad intelectual, global o por componentes; el rendimiento académico medido por las notas medias del nivel predecesor y las actitudes psicológicas que motivan al individuo a seguir o no estudios superiores. Esta motivación es el resultado de un conjunto de causas sociales y familiares ya mencionadas, pero también es efecto de la propia personalidad.

6. Por su parte, existen una serie de factores tecnológicos, caracterizados por la generación de nuevos avances científicos y, especialmente, por la difusión de nuevas tecnologías, lo que demanda de los titulados un alto grado de versatilidad y con capacidad de adaptarse rápidamente a los cambios.

7. Por último, y no menos relevantes, aparecen una serie de factores institucionales, que son aquellos que dependen de las características de los propios centros de enseñanza y que, progresivamente, han de adaptarse a las exigencias derivadas de la convergencia europea. Estos factores influyen más que en la demanda global, en la específica por centros o por tipos de estudios. Algunas de las características de 
las instituciones educativas que deben ejercer influencia sobre la demanda son: el nivel de calidad del centro, el nivel de prestigio (que no ha de coincidir necesariamente con el de la calidad), otros factores ambientales (localización, tamaño, etc.) y, fundamentalmente, la distancia entre la residencia del estudiante y el centro universitario.

Como se aprecia, uno de los elementos más relevantes que provocan que un individuo decida cursar estudios universitarios y que condicionan su estancia en las aulas es la motivación, también considerada en los estudios realizados por Valle, González y Cuevas (1997) en cuanto al logro de unas determinadas metas académicas y personales. La motivación significa movimiento, un movimiento con un propósito, que puede partir del propio individuo o ser inducido por estímulos del ambiente (Grzib, 2002). Estamos motivados cuando tenemos no sólo la necesidad, sino la voluntad de conseguir un objetivo, perseverando en el esfuerzo de ese logro durante el tiempo necesario. Ese movimiento está directamente relacionado con las actitudes, porque son pieza clave que guía el proceso de toma de decisiones, discriminando incluso entre lo que es o no importante. Las actitudes son las predisposiciones a responder de una determinada manera con reacciones favorables o desfavorables hacia algo. Las integran las opiniones o creencias, los sentimientos y las conductas, factores que a su vez se interrelacionan entre sí. Las opiniones son ideas que uno posee sobre un tema y no tienen por qué sustentarse en una información objetiva. Por su parte, los sentimientos son reacciones emocionales que se presentan ante un objeto, sujeto o grupo social. Finalmente, las conductas son tendencias a comportarse según opiniones o sentimientos propios. Las actitudes orientan los actos si las influencias externas sobre lo que se dice o hace tienen una mínima incidencia. También los orientan si la actitud tiene una relación específica con la conducta, a pesar de lo cual la evidencia confirma que, a veces, el proceso acostumbra a ser inverso y los actos no se corresponden, experimentándose una tensión denominada disonancia cognitiva. De este modo, el comportamiento del alumnado no es el resultado de una única actitud, sino que dependerá del conjunto de actitudes que le configuran en cada momento.

Considerando los distintos factores que afectan a la decisión tomada por un sujeto de cursar unos determinados estudios universitarios, los objetivos de este estudio se centran en advertir cuáles son las motivaciones y actitudes que guían este proceso y analizar su evolución a lo largo del tiempo que dura su carrera. 


\section{Método}

\section{Participantes}

La muestra ascendió a 800 estudiantes distribuidos en función las dos variables de estratificación empleadas, pertenencia a los primeros o últimos cursos, tal y como figura en la tabla 1. Cabe destacar que la mayor parte de alumnado se concentra en titulaciones pertenecientes a la rama Jurídico-Social (Magisterio, Psicopedagogía, Derecho, Empresariales, etc.).

Tabla 1: Muestra

\begin{tabular}{|l|c|c|c|c||}
\cline { 2 - 5 } \multicolumn{1}{c|}{} & \multicolumn{4}{c||}{ Curso } \\
\cline { 2 - 6 } \multicolumn{1}{c|}{} & \multicolumn{2}{c|}{ Primeros } & \multicolumn{2}{c||}{ Últimos } \\
\hline \hline Rama de especialización & f & \% & f & $\%$ \\
\hline \hline Ciencias de la Salud & 105 & 23.0 & 46 & 13.7 \\
\hline Experimentales & 58 & 12.6 & 15 & 4.3 \\
\hline Humanidades & 42 & 9.3 & 69 & 20.2 \\
\hline Jurídico-Sociales & 201 & 43.8 & 167 & 48.5 \\
\hline Técnicas & 52 & 11.2 & 45 & 13.3 \\
\hline \hline Total & $\mathbf{4 5 8}$ & $\mathbf{1 0 0}$ & $\mathbf{3 4 2}$ & $\mathbf{1 0 0}$ \\
\hline
\end{tabular}

\section{Instrumento}

A los de estudiantes se le aplicó un cuestionario formado por 32 ítems que hacen referencia a los motivos de elección de estudios, los objetivos de la educación superior, los derechos de los estudiantes universitarios y sus consiguientes deberes (ver anexo 1). Hemos verficado la calidad de este instrumento a través de un estudio de fiabilidad (análisis de consistencia interna) obteniendo un valor total de Alpha de $0.91(p<0,05)$ que nos confirma una consistencia aceptable del instrumento, por lo que podemos considerar que éste goza de las suficientes garantías para ser aplicado, analizado e interpretado

\section{Procedimiento}

Se ha llevado a cabo un diseño de investigación empírica no experimental, descriptivo y correlacional, utilizando los estudios de encuesta como base para la obtención de información. Debido a la imposibilidad de llevar a cabo un estudio longitudinal, hemos optado por una estrategia transversal en la que comparamos grupos de variables en estudiantes de nuevo ingreso y en estudiantes en el momento de concluir sus estudios.

Dada la particularidad de este trabajo, las variables que han sido seleccionadas para operativizar los objetivos, dan cuenta de dos de los elementos que condicionan el acceso y la 
permanencia en la Universidad: la motivación y las actitudes. Éstas han quedado definidas del siguiente modo:

- Variables motivacionales (motivos de elección de carrera). Con estos datos pretendemos conocer cuáles son los motivos que suscitaron al alumnado la realización de su titulación universitaria. Para ello, hemos tratado de advertir si esta decisión responde a gustos personales, facilidad, tradición familiar, profesión para la que capacita, oportunidades de empleo, imposibilidad de acceder a otra, y sin motivos aparentes.

- Actitudes del alumnado hacia la Universidad: con estas variables intentamos medir la opinión de los estudiantes encuestados con respecto a lo que representan y persiguen los estudios universitarios y, más concretamente, qué significa para ellos la educación superior como miembros del sistema en el que se encuentran inmersos (responsabilidades adquiridas, beneficios otorgados y contraprestaciones ofrecidas). Éstas se establecen en:

- Creencias sobre el contexto: grado de importancia otorgado a los Objetivos de la Educación Superior ${ }^{1}$.

- Concepción del papel del estudiante en la Universidad: consulta de los derechos y los deberes de los estudiantes por parte de los mismos y grado de importancia otorgada a esos derechos y deberes.

Este segundo conjunto de variables será aplicado a dos grupos muestrales diferentes. En primer lugar, obtendremos información del alumnado de nuevo ingreso para, posteriormente, llevar a cabo un estudio comparativo aplicando estas variables a una muestra representativa de estudiantes que están a punto de concluir sus estudios universitarios e ingresar en el mercado laboral.

Los análisis a realizar sobre cada una de estas variables se hará teniendo en cuenta el género de los alumnos, el curso de pertenencia (primeros y últimos) y la rama de especialización a la que pertenecen las titulaciones en las que se encuentran matriculados (Ciencias de la Salud, Humanidades, Jurídico-Sociales, Experimentales y Técnicas).

\section{Resultados}


La información obtenida tras la aplicación del instrumento y su posterior análisis se estructura en tres grandes apartados. En primer lugar, se exponen los datos referentes a los factores que motivan a los estudiantes a cursar estudios universitarios; en segundo lugar, se analizan las actitudes del alumnado recién llegado a la institución para, en tercer lugar, realizar un estudio comparativo con los estudiantes que cursan el último curso de su titulación sobre la base de esas actitudes.

\section{Motivos de elección de estudios}

De los diferentes motivos expuestos al alumnado encuestado, aquel que ha supuesto, con una frecuencia del 56.3\% (ver tabla 2) el más seleccionado a la hora de tomar la decisión de cursar una carrera universitaria es: Se trata de la carrera que más me gusta. Lo que indica un proceso basado en motivaciones personales.

Tabla 2: Motivos de elección de carrera

\begin{tabular}{|l|c|c||}
\hline Motivo de elección & f & \% \\
\hline \hline Me gusta & 450 & 56.3 \\
\hline Es fácil & 2 & 0.3 \\
\hline Tradición familiar & 3 & 0.4 \\
\hline Por la profesión & 137 & 17.1 \\
\hline Oportunidades empleo & 77 & 9.6 \\
\hline No puede otra & 56 & 7.0 \\
\hline Sin motivo & 50 & 6.3 \\
\hline Otro & 25 & 3.1 \\
\hline \hline Total & $\mathbf{8 0 0}$ & $\mathbf{1 0 0}$ \\
\hline \hline
\end{tabular}

Por su parte, un $17.1 \%$ afirmó cursar esos estudios por la profesión para la que capacitaban, lo que implica unas altas expectativas de futuro, no excluyente del primero de los motivos elegidos.

Atendiendo al género de los estudiantes, apenas encontramos variación alguna en los motivos. Únicamente señalar que los hombres difieren algo más, aunque no de forma significativa, que las mujeres al elegir su carrera motivados por las oportunidades de empleo que ésta conlleva (13.2\% frente a 7.5\%), motivo que indica unas altas expectativas laborales.

\footnotetext{
${ }^{1}$ Sobre la base de los objetivos formulados en la Ley de Reforma Universitaria (1983) y en la Ley Orgánica de Universidades (2001).
} 
Por su parte, en función del curso que realizan, los alumnos únicamente muestran diferencias significativas a favor del alumnado de primer curso, al hablar del motivo me gusta (ji cuadrado $=22.168, \mathrm{p}=0.02$ ). Este dato puede deberse a una mejor orientación hacia la Universidad, lo que facilita el proceso final de toma de decisiones.

Con respecto a la rama de especialización a la que pertenecen las titulaciones cursadas por los estudiantes, el motivo principal para todas ellas y estadísticamente significativo (n.s. $=0.001)$, al haber obtenido un valor de ji cuadrado=473.777 $(\mathrm{p}=0.000)$, es me gusta, por lo que las diferencias que puedan apreciarse entre el resto de los motivos no tienen ninguna validez en el ámbito estadístico.

Es relevante acercarse a los datos de la tabla 3, ya que proporciona información sobre la tendencia de los alumnos de las diferentes especialidades a la hora de decidirse por realizar una u otra titulación. Aclarado el motivo principal (me gusta), los alumnos de titulaciones de Ciencias de la Salud eligen su carrera por la profesión para la que capacita. Esto parece estar relacionado con la imagen social de la profesión a la que derivan sus estudios. Esta apreciación es similar para los estudiantes de titulaciones Jurídico-Sociales. Fundamentalmente, las profesiones médico y abogado tienen un prestigio social reconocido y una considerable compensación económica. Los alumnos de carreras Técnicas cursan su titulación motivados por las oportunidades de empleo que ofrecen, es decir, por las salidas profesionales que demanda actualmente el sistema productivo y su alta remuneración. Por último, podemos señalar que los estudiantes de titulaciones Experimentales manifiestan que cursan esas carreras porque no pudieron entrar en otras, no consideran que tengan prestigio social y buenas salidas profesionales.

Tabla 3: Motivo principal de elección de carrera en función de la rama de especialización

\begin{tabular}{|c|c|c|c|c|c|c|c|c|c|c|}
\hline \multirow[b]{3}{*}{ Motivo de elección } & \multicolumn{10}{|c|}{ Rama de especialización } \\
\hline & \multicolumn{2}{|c|}{$\begin{array}{l}\text { Ciencias } \\
\text { Salud }\end{array}$} & \multicolumn{2}{|c|}{$\begin{array}{c}\text { Experi- } \\
\text { mentales }\end{array}$} & \multicolumn{2}{|c|}{$\begin{array}{c}\text { Humani- } \\
\text { dades }\end{array}$} & \multicolumn{2}{|c|}{$\begin{array}{l}\text { Jurídico- } \\
\text { Sociales }\end{array}$} & \multicolumn{2}{|c|}{ Técnicas } \\
\hline & f & $\%$ & $\mathbf{f}$ & $\%$ & f & $\%$ & $\mathbf{f}$ & $\%$ & $\mathbf{f}$ & $\%$ \\
\hline Me gusta & 92 & 60.5 & 37 & 51.4 & 93 & 83.8 & 179 & 48.8 & 49 & 50.0 \\
\hline Es fácil & 0 & 0.0 & 0 & 0.0 & 0 & 0.0 & 2 & 0.5 & 0 & 0.0 \\
\hline Tradición familiar & 2 & 1.3 & 0 & 0.0 & 0 & 0.0 & 1 & 0.3 & 0 & 0.0 \\
\hline Por la profesión & 48 & 31.6 & 1 & 1.4 & 8 & 7.2 & 69 & 18.8 & 11 & 11.2 \\
\hline Oportunidades empleo & 2 & 1.3 & 6 & 8.3 & 2 & 1.8 & 36 & 9.8 & 31 & 31.6 \\
\hline No puede otra & 2 & 1.3 & 22 & 30.6 & 1 & 0.9 & 29 & 7.9 & 2 & 2.0 \\
\hline Sin motivo & 5 & 3.3 & 6 & 8.3 & 6 & 5.4 & 29 & 7.9 & 4 & 4.1 \\
\hline
\end{tabular}




\begin{tabular}{|l|c|c||c|c||c|c||c|c||c|c|}
\hline Otro & 1 & 0.7 & 0 & 0.0 & 1 & 0.9 & 22 & 6.0 & 1 & 1.0 \\
\hline Total & $\mathbf{1 5 2}$ & $\mathbf{1 0 0}$ & $\mathbf{7 2}$ & $\mathbf{1 0 0}$ & $\mathbf{1 1 1}$ & $\mathbf{1 0 0}$ & $\mathbf{3 6 7}$ & $\mathbf{1 0 0}$ & $\mathbf{9 8}$ & $\mathbf{1 0 0}$ \\
\hline
\end{tabular}

Actitudes del alumnado de nuevo ingreso

A continuación, recogemos todos aquellos datos referentes a las actitudes del alumnado de nuevo ingreso. Bajo esta denominación definimos aquellas variables que determinan los fines de una institución universitaria desde la opinión de los estudiantes (creencias sobre el contexto, a través del grado de importancia otorgada a los objetivos de la Educación Superior), así como la concepción que da el propio alumno de sí, como parte integrante de la institución universitaria a través de sus derechos y deberes (consulta e importancia otorgada). Se trata únicamente de recoger las opiniones para así, considerar la actitud inicial con la que llega el alumno a la Universidad.

\section{Creencias sobre el contexto}

Respecto de la Importancia otorgada a los Objetivos de la Educación Superior, los estudiantes manifiestan que el más importante es capacitar a los individuos para desempeñar las funciones que exige la división del trabajo (ver tabla 4). Es interesante ver cómo son considerados en su gran mayoría, todos los objetivos, como muy importantes, dando mayor valor a aquellos que tienen que ver directamente con la inserción en el mercado laboral. Esta tendencia se mantiene si diferenciamos a los alumnos en función del género, no así en cuanto hablamos de la rama de especialización.

Tabla 4: Objetivos de la Educación Superior

\begin{tabular}{|l||c||c||c|c|c|c|c||c||}
\hline Objetivos & $\overline{\mathrm{X}}$ & $\mathrm{S}_{\mathrm{X}}$ & $\begin{array}{c}\text { sin impor- } \\
\text { tancia (\%) }\end{array}$ & $\begin{array}{c}\text { poco } \\
\text { importante } \\
(\%)\end{array}$ & $\begin{array}{c}\text { importante } \\
(\%)\end{array}$ & $\begin{array}{c}\text { bastante } \\
\text { importante } \\
(\%)\end{array}$ & $\begin{array}{c}\text { muy impor- } \\
\text { tante (\%) }\end{array}$ & $\mathrm{N}$ \\
\hline \hline 1. Cultura y valores & 3.54 & 1.13 & 4.4 & 12.3 & 33.6 & 24.2 & 25.5 & 455 \\
\hline 2. Ampliar conocimiento & 3.92 & 0.99 & 1.5 & 6.1 & 25.5 & 32.5 & 34.3 & 458 \\
\hline 3. Transmisión crítica & 3.71 & 1.02 & 2 & 10.3 & 27.8 & 34.8 & 25.2 & 457 \\
\hline 4. División del trabajo & 3.94 & 1.08 & 2 & 8.8 & 22.8 & 26.3 & 40.3 & 457 \\
\hline 5. Mejora sistema educativo & 3.75 & 1.11 & 3.9 & 9 & 26.5 & 29.8 & 30.9 & 457 \\
\hline 6. Actividad intelectual & 3.86 & 1.08 & 2.9 & 9.4 & 20.6 & 32.9 & 34.2 & 456 \\
\hline 7. Asesorar a la sociedad & 3.73 & 1.02 & 1.8 & 11 & 25.7 & 35.7 & 25.9 & 456 \\
\hline 8. Cooperación desarrollo & 3.81 & 1.07 & 3.3 & 7.4 & 26.4 & 30.3 & 32.5 & 458 \\
\hline
\end{tabular}

La aplicación de un análisis de varianza de un factor conlleva la existencia de diferencias significativas en cuatro de los ocho objetivos planteados: en primer lugar y con un 
valor de $\mathrm{F}=4.873$ ( $\mathrm{p}=0.001$ ), el primer objetivo (transmitir la cultura y los valores de la sociedad) asume diferencias entre las titulaciones de las áreas Técnicas y Jurídico-Sociales, a favor de estas últimas (n.s.=0.01). En segundo lugar, el objetivo ampliar y avanzar en el conocimiento a través de la investigación en todas las ramas del saber, admite diferencias a favor de las titulaciones del área de Salud, frente a carreras de las áreas de Humanidades y Jurídico-Sociales. En tercer lugar, y con un valor de $\mathrm{F}=2.527(\mathrm{p}=0.04)$ el objetivo definido como transmitir de forma crítica el saber a través de la actividad docente y la discusión crítica muestra diferencias a favor de titulaciones de la rama de Salud frente a carreras de la rama Experimental y Jurídico-Social (n.s.=0.05). Por último, el cuarto objetivo que muestra diferencias significativas $(\mathrm{n} . \mathrm{s} .=0.01)$ consiste en capacitar a los individuos para desempeñar con las funciones que exige la división del trabajo. Con un valor de $\mathrm{F}=4.657$ ( $\mathrm{p}=0.001)$, las diferencias han sido notables a favor de titulaciones del área de Salud frente a titulaciones del área Humanidades y a favor de carreras Técnicas en detrimento, nuevamente, de las Humanidades.

Concepción del papel del alumno en la Universidad

Esta variable refleja la ausencia casi total por parte de los estudiantes en lo que se refiere a la consulta de sus derechos y deberes. Un $75.8 \%$ nunca ha consultado sus derechos y un $80.1 \%$ nunca ha consultado sus deberes (ver tabla 5 ).

Tabla 5: Consulta de derechos y deberes por parte de los estudiantes universitarios

\begin{tabular}{|l||c||c||c|c|c||c||}
\hline Derechos/Deberes & $\overline{\mathrm{X}}$ & $\mathrm{S}_{\mathrm{x}}$ & $\begin{array}{c}\text { nunca } \\
(\%)\end{array}$ & $\begin{array}{c}\text { en varias ocasiones } \\
(\%)\end{array}$ & $\begin{array}{c}\text { en múltiples ocasio- } \\
\text { nes (\%) }\end{array}$ & $\mathrm{N}$ \\
\hline \hline 1. Consulta derechos & 1.26 & 0.47 & 75.8 & 22.6 & 1.5 & 455 \\
\hline 2. Consulta deberes & 1.21 & 0.44 & 80.1 & 18.5 & 1.3 & 453 \\
\hline
\end{tabular}

Por su parte, un estudio inferencial a través de la prueba de t para muestras independientes, no revela diferencias estadísticamente significativas tomando como grupos de análisis el género de los estudiantes y la rama de especialización a la que pertenecen sus estudios.

Aún sabiendo que los estudiantes apenas han consultado sus derechos, al ofrecerles la oportunidad de valorarlos, advierten que el más importante es recibir las enseñanzas teóricas y prácticas correspondientes a la titulación elegida, seguido de su derecho a recibir in- 
formación sobre becas y ayudas al estudio y formar parte de las comisiones que las otorguen, en los valores señalados en la tabla 6 .

Tabla 6: Derechos de los estudiantes universitarios

\begin{tabular}{|c|c|c|c|c|c|c|c|c|}
\hline Derechos & $\bar{X}$ & $\mathrm{~S}_{\mathrm{X}}$ & $\begin{array}{l}\text { sin impor- } \\
\text { tancia (\%) }\end{array}$ & $\begin{array}{c}\text { poco } \\
\text { importante } \\
(\%)\end{array}$ & $\begin{array}{l}\text { importante } \\
(\%)\end{array}$ & $\begin{array}{l}\text { bastante } \\
\text { importante } \\
(\%)\end{array}$ & $\begin{array}{c}\text { muy impor- } \\
\text { tante }(\%)\end{array}$ & $\mathrm{N}$ \\
\hline 1. Recibir enseñanzas & 4.7 & 0.62 & 0.2 & 0.4 & 6.3 & 15.2 & 77.8 & 460 \\
\hline 2. Elegir profesor & 3.63 & 1.12 & 3.7 & 12 & 29.3 & 27.6 & 27.4 & 460 \\
\hline 3. Participar actividades & 3.82 & 0.93 & 1.7 & 5.2 & 27.9 & 39.4 & 25.7 & 459 \\
\hline 4. Tutorías & 4.1 & 0.96 & 1.5 & 4.4 & 19.6 & 31.6 & 42.9 & 459 \\
\hline 5. Oferta docente y exámenes & 4.47 & 0.76 & 0.2 & 1.1 & 12 & 25.2 & 61.5 & 460 \\
\hline 6. Valoración rendimiento & 4.4 & 0.81 & 0.9 & 1.1 & 12.2 & 28.8 & 57 & 458 \\
\hline 7. Programas gratis & 4.36 & 0.94 & 1.8 & 3.3 & 12.1 & 22.5 & 60.4 & 454 \\
\hline 8. Información becas y ayudas & 4.5 & 0.76 & 0 & 1.3 & 12.4 & 20.9 & 65.4 & 459 \\
\hline 9. Instalaciones adecuadas & 4.46 & 0.78 & 0 & 2.6 & 9.8 & 27 & 60.7 & 460 \\
\hline 10. Participación gobierno & 3.49 & 1.06 & 3 & 13.3 & 36.3 & 26.5 & 20.9 & 460 \\
\hline 11. Asociarse libremente & 3.81 & 1.11 & 3.5 & 8.3 & 27.1 & 26.2 & 34.9 & 458 \\
\hline 12. Información derechos & 4.2 & 0.9 & 0.2 & 4.1 & 18.9 & 28.7 & 48 & 460 \\
\hline
\end{tabular}

La comparación de esta variable entre hombres y mujeres refleja diferencias significativas a favor de las mujeres (n.s. $=0.05)$, tras la realización de una prueba de t para muestras independientes, a la hora de otorgar valor a los derechos 4,10 y 12, cuyos valores t son -3.105 $(\mathrm{p}=0.002),-2.908(\mathrm{p}=0.004)$ y $-2.473(\mathrm{p}=0.014)$ respectivamente. Estos derechos están relacionados con los sistemas de tutorías, la participación en el gobierno y representación de la Universidad y la recepción de información sobre el funcionamiento de esta institución.

Tras la aplicación del análisis de varianza de un factor (n.s. $=0.05)$, tomando como variable independiente la rama de especialización, podemos apreciar que existen las siguientes diferencias significativas:

- Con un valor de $\mathrm{F}=2.864$ ( $\mathrm{p}=0.023$ ), el alumnado de las titulaciones pertenecientes a las especialidades de Salud, Jurídico-Sociales y Técnicas, frente a titulaciones Experimentales, considera que un derecho fundamental como estudiantes universitarios consiste en elegir el profesor que imparta la docencia y evaluar su rendimiento.

- Por su parte, son los estudiantes de titulaciones de la rama Técnica quienes consideran que es un derecho fundamental, en detrimento de las titulaciones del área Experimental, ser asistidos mediante un sistema de tutorías ( $\mathrm{F}=2.8$, $\mathrm{p}=0.026)$. 
- En lo que respecta al derecho a recibir una valoración objetiva de su rendimiento académico y conocer los criterios de valoración del mismo, las diferencias encontradas son favorables a los estudiantes de carreras de Ciencias de la Salud y Técnicas en perjuicio de las Experimentales $(\mathrm{F}=4.002, \mathrm{p}=0.003)$.

- El alumnado considera un derecho fundamental recibir gratuitamente los programas de cada asignatura. Sin embargo esta importancia es prioritaria para los estudiantes de carreras Técnicas sobre titulaciones de la rama Experimental, de Humanidades y Jurídico-Sociales; así como en carreras del área de Salud sobre titulaciones del área Experimental.

- Por último, la valoración realizada por los estudiantes de carreras del área Jurídico-Social es diferente a la realizada por los estudiantes de Humanidades, siendo esta última más baja $(\mathrm{F}=3.686, \mathrm{p}=0.004)$ al manifestar como derecho fundamental de todo alumno universitario asociarse libremente.

Al igual que en el caso anterior, el alumnado valoró los diferentes deberes que ha de asumir tras su ingreso en la institución universitaria. Sin lugar a dudas el más importante es el respeto de las normas y del patrimonio de la Universidad, así como la asunción de responsabilidades de los puestos para los que sean elegidos. Esto parece estar muy relacionado con la valoración de los derechos anteriores. La tabla que se muestra a continuación revela los diferentes rangos de importancia otorgada a esta variable (ver tabla 7).

Tabla 7: Deberes de los estudiantes universitarios

\begin{tabular}{|l||c||c||c|c|c|c|c||c||}
\hline Deberes & $\overline{\mathrm{X}}$ & $\mathrm{S}_{\mathrm{X}}$ & $\begin{array}{c}\text { sin impor- } \\
\text { tancia (\%) }\end{array}$ & $\begin{array}{c}\text { poco } \\
\text { importante } \\
(\%)\end{array}$ & $\begin{array}{c}\text { importante } \\
(\%)\end{array}$ & $\begin{array}{c}\text { bastante } \\
\text { importante } \\
(\%)\end{array}$ & $\begin{array}{c}\text { muy impor- } \\
\text { tante (\%) }\end{array}$ & $\mathrm{N}$ \\
\hline \hline 1. Respetar normas & 4.41 & 0.8 & 0.7 & 1.5 & 11.7 & 28.5 & 57.6 & 460 \\
\hline 2. Trabajo propio & 4.18 & 0.89 & 1.5 & 1.3 & 19.2 & 34 & 44 & 459 \\
\hline 3. Asumir responsabilidades & 4.22 & 0.94 & 1.3 & 3 & 18.3 & 27.6 & 49.8 & 460 \\
\hline 4. Cooperar mejora & 3.95 & 0.9 & 0.7 & 4.8 & 24.4 & 38.8 & 31.4 & 459 \\
\hline 5. Cooperar logro objetivos & 3.81 & 1.01 & 2.6 & 5.9 & 28.3 & 34.1 & 29.1 & 460 \\
\hline
\end{tabular}


Existen diferencias estadísticamente significativas (n.s.=0.05) en la importancia otorgada a todos los deberes a excepción del primero, procedentes de un estudio t para muestras independientes en el que se ha tomado como variable independiente el género de los estudiantes. Estas demuestran que hombres y mujeres valoran de diferente manera estos cuatros deberes, aceptando un nexo común el respeto de las normas. Las diferencias obtenidas son favorables, en todos los casos, a las mujeres.

Sin embargo, la búsqueda de diferencias en función de la rama de especialización no ha arrojado valoraciones significativas tras la realización de un análisis de varianza para un factor.

\section{Actitudes del alumnado de último curso hacia la Universidad}

Teniendo en cuenta que este grupo de variables fue analizado a partir de las opiniones expuestas por los alumnos de nuevo ingreso, estudiaremos las creencias sobre el contexto y la concepción de estudiante universitario de los alumnos que cursan último curso de carrera. En un intento por estudiar la evolución de estas actitudes por parte del alumnado a lo largo de sus estudios, hemos llevado a cabo un estudio inferencial a través de la prueba t de Student a este conjunto de variables, tomando como criterio de clasificación el curso al que pertenecen los estudiantes encuestados.

\section{Creencias sobre el contexto}

Los alumnos y alumnas de último año consideran que la Universidad tiene como principal objetivo ampliar y avanzar en el conocimiento a través de la investigación en todas las ramas de la cultura, la ciencia y la técnica. Al igual que el resto de sus compañeros, consideran que todos los objetivos planteados son importantes, destacando aquellos relacionados directamente con la formación integral (ver tabla 8).

Tabla 8: Objetivos de la Educación Superior

\begin{tabular}{|l||c||c||c|c|c|c|c||c||}
\hline Objetivos & $\overline{\mathrm{X}}$ & $\mathrm{S}_{\mathrm{X}}$ & $\begin{array}{c}\text { sin impor- } \\
\text { tancia (\%) }\end{array}$ & $\begin{array}{c}\text { poco } \\
\text { importante } \\
(\%)\end{array}$ & $\begin{array}{c}\text { importante } \\
(\%)\end{array}$ & $\begin{array}{c}\text { bastante } \\
\text { importante } \\
(\%)\end{array}$ & $\begin{array}{c}\text { muy impor- } \\
\text { tante (\%) }\end{array}$ & $\mathrm{N}$ \\
\hline \hline 1. Cultura y valores & 3.43 & 1.21 & 7 & 15.4 & 29.9 & 22.9 & 24.9 & 345 \\
\hline 2. Ampliar conocimiento & 3.83 & 1.15 & 3.2 & 12.2 & 20.1 & 27.9 & 36.6 & 344 \\
\hline 3. Transmisión crítica & 3.59 & 1.19 & 4.4 & 16.3 & 24.4 & 25.6 & 29.4 & 344 \\
\hline 4. División del trabajo & 3.67 & 1.21 & 5.8 & 12.2 & 22.7 & 27.3 & 32 & 344 \\
\hline 5. Mejora sistema educativo & 3.45 & 1.19 & 5.6 & 16.7 & 30.4 & 21.9 & 25.4 & 342 \\
\hline
\end{tabular}




\begin{tabular}{||l||c||c||c|c|c|c|c||c||}
\hline 6. Actividad intelectual & 3.78 & 1.15 & 2 & 15.5 & 204 & 26.8 & 35.3 & 343 \\
\hline 7. Asesorar a la sociedad & 3.53 & 1.1 & 3.5 & 14.7 & 30.2 & 28.4 & 23.2 & 341 \\
\hline 8. Cooperación desarrollo & 3.7 & 1.19 & 4.9 & 11.9 & 24.1 & 26.2 & 32.8 & 344 \\
\hline
\end{tabular}

Realizando una diferenciación en las actitudes por género (prueba t para muestras independientes, n.s. $=0.05$ ), nos damos cuenta que para las mujeres el objetivo más importante que ha de cumplir la Universidad es contribuir a la mejora del sistema educativo $(\mathrm{t}=-2.482$, $\mathrm{p}=0.005$ ). Para los hombres, sin embargo, adquiere mayor relevancia cooperar con el desarrollo científico, técnico, cultural y social de todos los pueblos $(\mathrm{t}=2.384, \mathrm{p}=0.018)$ y asesorar a científica, técnica y culturalmente a la sociedad de modo que se satisfagan sus necesidades $(\mathrm{t}=2.004, \mathrm{p}=0.046)$.

Por su parte, la realización de una análisis de varianza para un factor, tomando como variable dependiente la rama de especialización de los estudios a punto de concluir, ofrece las diferencias significativas (n.s. $=0.05)$ que se señalan a continuación:

- Los alumnos de Humanidades, frente a sus compañeros de las áreas JurídicoSocial y Experimental, consideran que lo más importante es transmitir la cultura y los valores de la sociedad $(\mathrm{F}=5.897, \mathrm{p}=0.000)$, estimular la actividad intelectual en todos los ámbitos $(\mathrm{F}=3.334, \mathrm{p}=0.011)$, y frente a sus compañeros tanto de las áreas Jurídico-Sociales y Técnicas, es contribuir a la mejora del sistema educativo $(\mathrm{F}=2.739, \mathrm{p}=0,029)$.

- Los estudiantes de carreras de las áreas de Humanidades y Técnicas, a diferencia de sus compañeros de carreras del área Jurídico-Sociales, creen que es importante ampliar y avanzar en el conocimiento a través de la investigación en todas las ramas de la cultura, la ciencia y la técnica $(\mathrm{F}=5.142, \mathrm{p}=0.000)$, así como cooperar con el desarrollo científico, técnico, cultural y social de todos los pueblos $(\mathrm{F}=3.258, \mathrm{p}=0.012)$.

Por último, tratando de estudiar la evolución que ha sufrido la opinión que guardan los estudiantes con respecto a los fines que ha de cumplir la Universidad, un estudio inferencial (prueba t para muestras independientes, n.s. $=0.01$ ) nos permite advertir que, únicamente los alumnos de primer curso consideran más importante capacitar a los individuos para desempeñar las funciones que exige la división del trabajo $(\mathrm{t}=3.441, \mathrm{p}=0.001)$ y asesorar a la sociedad de modo que se satisfagan sus necesidades $(\mathrm{t}=2.875, \mathrm{p}=0.004)$. 


\section{Concepción del papel del estudiante en la Universidad}

Al igual que en el caso de los estudiantes de primer curso, y no existiendo diferencia significativa alguna tras la aplicación de una prueba t para muestras independientes, un $70.7 \%$ de los estudiantes de últimos cursos nunca ha consultado sus derechos y un $77 \%$ nunca ha consultado sus deberes, ya se trate de hombres o mujeres o de cualquier titulación (ver tabla 9).

Tabla 9: Consulta de derechos y deberes por parte de los estudiantes universitarios

\begin{tabular}{|l||c||c||c|c|c||c||}
\hline \hline Derechos/Deberes & $\overline{\mathrm{X}}$ & $\mathrm{S}_{\mathrm{X}}$ & nunca (\%) & $\begin{array}{c}\text { en varias ocasiones } \\
(\%)\end{array}$ & $\begin{array}{c}\text { en múltiples ocasio- } \\
\text { nes (\%) }\end{array}$ & $\mathrm{N}$ \\
\hline \hline 1. Consulta derechos & 1.31 & 0.5 & 70.7 & 27.5 & 1.7 & 345 \\
\hline 2. Consulta deberes & 1.25 & 0.47 & 77 & 21.3 & 1.7 & 434 \\
\hline
\end{tabular}

A pesar de esta premisa, este grupo de alumnos, al igual que en el caso de los de nuevo ingreso (ver tabla 10), considera que su derecho más importante como estudiantes universitarios consiste en recibir las enseñanzas teóricas y prácticas correspondientes a la titulación elegida, seguido de su derecho a recibir información sobre becas y ayudas al estudio. Sin embargo, mientras que los estudiantes de primer año consideran significativamente más importante recibir enseñanzas $(\mathrm{t}=2.268, \mathrm{p}=0.024)$ y recibir gratuitamente los programas de las asignaturas $(\mathrm{t}=3.791, \mathrm{p}=0)$, el alumnado de último año considera significativamente más relevante ser asistidos mediante un buen sistema de tutorías $(\mathrm{t}=-2.136, \mathrm{p}=0.033)$ y participar en los órganos de gobierno y representación de la Universidad $(\mathrm{t}=-2.098, \mathrm{p}=0.036)$.

Tabla 10: Derechos de los estudiantes universitarios

\begin{tabular}{|c|c|c|c|c|c|c|c|c|}
\hline Derechos & $\bar{X}$ & $\mathrm{~S}_{\mathrm{X}}$ & $\begin{array}{l}\text { sin impor- } \\
\text { tancia (\%) }\end{array}$ & $\begin{array}{c}\text { poco } \\
\text { importante } \\
(\%)\end{array}$ & $\begin{array}{l}\text { importante } \\
(\%)\end{array}$ & $\begin{array}{c}\text { bastante } \\
\text { importante } \\
(\%)\end{array}$ & $\begin{array}{c}\text { muy impor- } \\
\text { tante }(\%)\end{array}$ & $\mathrm{N}$ \\
\hline 1. Recibir enseñanzas & 4.59 & 0.68 & 0 & 0.6 & 9.5 & 19.9 & 59.9 & 346 \\
\hline 2. Elegir profesor & 3.67 & 1.08 & 2.6 & 10.2 & 33.8 & 23.9 & 29.4 & 343 \\
\hline 3. Participar actividades & 3.92 & 0.9 & 0.6 & 4.1 & 29 & 35.7 & 30.7 & 345 \\
\hline 4. Tutorías & 4.24 & 0.85 & 0.6 & 1.7 & 18.3 & 32.3 & 47.1 & 344 \\
\hline 5. Oferta docente y exámenes & 4.38 & 0.85 & 0.3 & 3.2 & 13.1 & 25 & 58.4 & 344 \\
\hline 6. Valoración rendimiento & 4.4 & 0.77 & 0 & 1.5 & 13.1 & 29.7 & 55.7 & 343 \\
\hline 7. Programas gratis & 4.09 & 1.04 & 0.6 & 8.8 & 19.6 & 23.1 & 48 & 342 \\
\hline 8. Información becas y ayudas & 4.45 & 0.75 & 0 & 1.7 & 10.5 & 28.5 & 59.3 & 344 \\
\hline 9. Instalaciones adecuadas & 4.37 & 0.8 & 0 & 1.7 & 14.8 & 28.7 & 54.8 & 345 \\
\hline 10. Participación gobierno & 3.64 & 1.04 & 2.6 & 9.9 & 33.3 & 29.3 & 24.9 & 345 \\
\hline 11. Asociarse libremente & 3.68 & 1.14 & 3.5 & 11.4 & 30.8 & 22 & 32.3 & 341 \\
\hline 12. Información derechos & 4.18 & 0.88 & 0.3 & 2 & 23.5 & 27.5 & 46.7 & 345 \\
\hline
\end{tabular}


La comparación de esta variable entre hombres y mujeres refleja diferencias significativas (n.s. $=0.05)$ a favor de las mujeres, tras la realización de una diferencia de medias para muestras independientes, a la hora de otorgar valor a los derechos $4(\mathrm{t}=-2.547, \mathrm{p}=0.012), 10$ $(\mathrm{t}=-2.766, \mathrm{p}=0.006)$ y $12(\mathrm{t}=-2.201, \mathrm{p}=0.028)$, al igual que en el caso del alumnado de primer curso, sumando a ellos el derecho $8(\mathrm{t}=-2.355, \mathrm{p}=0.019)$. Estos derechos se refieren al sistema de tutorías, la participación en el gobierno universitario, la recepción de información sobre el funcionamiento interno de la institución, así como sobre becas y ayudas al estudio.

La realización de una análisis de varianza para un factor tomando como variable de clasificación la rama de especialización, aporta una serie de diferencias significativas (n.s. $=0.05)$ a favor de los alumnos de Ciencias de la Salud al valorar su derecho a elegir profesor $(\mathrm{F}=5.445, \mathrm{p}=0.000)$, a recibir una valoración objetiva de su rendimiento $(\mathrm{F}=5.364$, $\mathrm{p}=0.000)$, a disponer de unas instalaciones adecuadas $(\mathrm{F}=2.614, \mathrm{p}=0.035)$ y a participar en los órganos de gobierno y representación $(\mathrm{F}=5.106, \mathrm{p}=0.001)$. Igualmente se han apreciado diferencias favorables en estudiantes de titulaciones del área Técnica, al valorar más positivamente el derecho a recibir enseñanzas $(\mathrm{F}=2.794, \mathrm{p}=0.026)$ que sus compañeros de titulaciones del área Jurídico-Social.

A la hora de ofrecer una valoración sobre sus deberes como estudiantes universitarios consideran, a punto de concluir sus estudios, que el más importante es el respeto de las normas y del patrimonio de la Universidad, así como la asunción de responsabilidades de los puestos para los que sean elegidos (ver tabla 11).

Tabla 11: Deberes de los estudiantes universitarios

\begin{tabular}{|l||c||c||c|c|c|c|c||c||}
\hline \hline Deberes & $\overline{\mathrm{X}}$ & $\mathrm{S}_{\mathrm{X}}$ & $\begin{array}{c}\text { sin impor- } \\
\text { tancia (\%) }\end{array}$ & $\begin{array}{c}\text { poco } \\
\text { importante } \\
(\%)\end{array}$ & $\begin{array}{c}\text { importante } \\
(\%)\end{array}$ & $\begin{array}{c}\text { bastante } \\
\text { importante } \\
(\%)\end{array}$ & $\begin{array}{c}\text { muy impor- } \\
\text { tante (\%) }\end{array}$ & $\mathrm{N}$ \\
\hline \hline 1. Respetar normas & 4.45 & 0.73 & 0.3 & 0.6 & 17.7 & 31 & 57.4 & 345 \\
\hline 2. Trabajo propio & 4.22 & 0.84 & 1.2 & 1.2 & 16.6 & 37.2 & 43.9 & 344 \\
\hline 3. Asumir responsabilidades & 4.29 & 0.82 & 0.6 & 0.6 & 17.4 & 31.1 & 50 & 344 \\
\hline 4. Cooperar mejora & 4.01 & 0.89 & 0.3 & 0.3 & 25 & 35.5 & 35.2 & 344 \\
\hline 5. Cooperar logro objetivos & 3.76 & 1.06 & 2.9 & 2.9 & 25.9 & 32.9 & 28.9 & 343 \\
\hline
\end{tabular}

Por otra parte, se aprecia la misma valoración que en el caso de los alumnos de primer curso, no advirtiendo diferencias significativas algunas tras la realización de una prueba $t$ 
para muestras independientes, tomando como criterio de valoración el nivel académico en curso.

Si realizamos una estudio de la valoración de estos deberes en función del género de los estudiantes, apreciamos diferencias significativas, tras la realización de un estudio t para muestras independientes (n.s. $=0.05$ ), a favor de las mujeres, quienes dotan de mayor importancia a su deber de cooperar con el resto de la comunidad universitaria en el buen funcionamiento de la Universidad y en la mejora de sus servicios $(\mathrm{t}=-3.46, \mathrm{p}=0.001)$, asumir las responsabilidades de los puestos para los que sean elegidos $(\mathrm{t}=-2.564, \mathrm{p}=0.011)$ y cooperar para conseguir los objetivos de la institución $(\mathrm{t}=-2.343, \mathrm{p}=0.013)$.

A diferencia de sus compañeros de primer curso, sí se encontraron diferencias significativas en lo que respecta a la valoración de sus deberes, teniendo en cuenta el tipo de titulación en curso. El estudio del análisis de varianza de un factor $(\mathrm{n} . \mathrm{s} .=0.05)$ reveló los siguientes resultados:

- Los estudiantes de titulaciones del área de Ciencias de la Salud, a diferencia del alumnado de titulaciones pertenecientes a la rama Jurídico-Social, consideran importantes sus deberes de asunción de las responsabilidades de los puestos para los que sean elegidos $(\mathrm{F}=4.241, \mathrm{p}=0.002)$ y el respeto de las normas y del patrimonio de la Universidad $(\mathrm{F}=3.852, \mathrm{p}=0.004)$.

- En otro sentido, estos mismos alumnos de Ciencias de la Salud, a diferencia de sus compañeros de titulaciones del área Técnica, valoran más positivamente su deber de cooperar con el resto de la comunidad universitaria en el buen funcionamiento de la Universidad y en la mejora de sus servicios $(\mathrm{F}=3.275, \mathrm{p}=0.012)$.

\section{Discusión}

Una recapitulación de lo expresado hasta este momento, revela que los motivos que guían al grupo encuestado en relación con la decisión de matricularse en sus titulaciones se caracterizan por sus gustos personales, siendo la carrera elegida su titulación preferida. Un porcentaje más reducido argumenta que el motivo por el cual han elegido esa carrera es por la profesión para la que capacita, factor de producto relacionado con el acceso al mundo laboral y la integración en la vida social. 
Haciendo referencia a las actitudes del alumnado de nuevo ingreso, estos consideran que el objetivo más importante que ha de cubrir este nivel educativo es capacitar a los individuos para desempeñar las funciones que exige la división del trabajo. Se trata de una actitud de futuro hacia el aprovechamiento funcional de los estudios elegidos. Hay que señalar que las mujeres advierten necesaria la transmisión de la cultura y los valores de la sociedad, así como la contribución de la mejora del sistema educativo. Al mismo tiempo, es importante destacar una ausencia casi total en la consulta de sus derechos y deberes como estudiantes universitarios. Aún así, creen que sus derechos adquiridos han de centrarse principalmente en recibir las enseñanzas teóricas y prácticas correspondientes a la titulación elegida, recibir información sobre becas y ayudas al estudio y formar parte de las comisiones que las otorgan, mientras que sus deberes más importantes son respetar las normas y el patrimonio de la Universidad, así como asumir las responsabilidades de los puestos para los que sean elegidos. Se trata de elementos relacionados con el fin práctico de los estudios, el funcionamiento interno de la Universidad y las posibilidades de participación que en ella se ofrecen.

En lo que respecta a las actitudes hacia la Universidad ya manifestadas por el alumnado de nuevo ingreso, podemos decir que, los alumnos de último curso consideran que la Universidad ha de ampliar y avanzar en el conocimiento a través de la investigación en todas las ramas de la cultura, la ciencia y la técnica. Hay que destacar que de nuevo, las mujeres de último año de carrera son conscientes de que la educación superior ha de contribuir a la mejora del sistema educativo, mientras que los hombres revelan, en este caso, la necesidad de asesorar a la sociedad de modo que se satisfagan sus necesidades y cooperar con el desarrollo integral de todos los pueblos. A pesar de ello, se encuentra de nuevo una ausencia casi total en la consulta de sus derechos y sus deberes. Sin embargo, los derechos y deberes más importantes coinciden con los expresados por sus compañeros de nuevo ingreso: recibir las enseñanzas teóricas y prácticas correspondientes a la titulación elegida, recibir información sobre becas y ayudas al estudio y formar parte de las comisiones que las otorguen, respetar las normas y el patrimonio de la Universidad y asumir las responsabilidades de los puestos para los que sean elegidos. Hay que destacar que el alumnado de último año considera que es un derecho relevante ser asistidos mediante un buen sistema de tutorías y participar en los órganos de gobierno y representación.

Con este estudio hemos podido comprobar la existencia de una serie de motivos patentes en el alumnado de nuestra muestra que condicionan la elección de los estudios universi- 
tarios y que llegan a configurar una serie de actitudes que apenas evolucionan a lo largo de su vida estudiantil. Es por ello, que podemos avanzar que estos dos elementos (motivación y actitudes) son claves en el desarrollo de la formación universitaria y componentes básicos a tener en cuenta a la hora de propiciar una Universidad de calidad.

\section{Referencias}

ANECA (2004). Los españoles y la Universidad. Madrid: ANECA.

Bricall, J.M. (2000). Universidad dosmil. Madrid: CRUE.

Cofer, C.N. y Appely, M.H. (1993). Psicología de la motivación. México: Trillas.

Gámez, E. y Marrero, H. (2003). Metas y motivos de elección de la carrera universitaria: un estudio comparativo entre Psicología, derecho y biología. Anales de Psicología, 19 (1), 121-131.

González, M.J. (1999). La Universidad del Siglo XXI. Libertad, competencia y calidad. Madrid: Círculo de Empresarios.

Grzib, G. (2002). Bases cognitivas y conductuales de la motivación y la emoción. Madrid: Centro de Estudios Ramón Areces.

Ley Orgánica 11/1983, de 25 de agosto, de Reforma Universitaria (Boletín Oficial del Estado número 209, de 1 de septiembre de 1983).

Mora Ruiz, J.G. (1990). La demanda de la educación superior. Madrid: Consejo de Universidades.

Ley Orgánica 6/2001, de 21 de diciembre, de Universidades (Boletín Oficial del Estado número 307, de 24 de diciembre de 2001).

Nunnally, J.C. (1978). Psycometric Theory. Nueva York: McGraw-Hill.

Valle, A. et al.(1999). Un modelo causal sobre los o cognitivo-motivacionales del rendimiento académico. Revista de Psicología General y Aplicada, 52 (4), 499-519.

Valle, A.; González, R. y Cuevas, L.M. (1997). Patrones motivacionales en estudiantes universitarios: características diferenciales. Revista de Investigación Educativa, 15 (1), 125-146. 
ANEXO 1

\section{CUESTIONARIO}

\section{A. CARACTERÍSTICAS DE LOS ALUMNOS}

1. Curso: $\square 1^{\circ} \quad \square 2^{\circ} \quad \square 3^{\circ} \quad \square 4^{\circ} \quad \square 5^{\circ} \quad \square 6^{\circ}$

2. Titulación en curso:

3. Sexo: $\square$ Hombre $\square$ Mujer

4. Motivo principal de elección de carrera: (elige sólo uno)

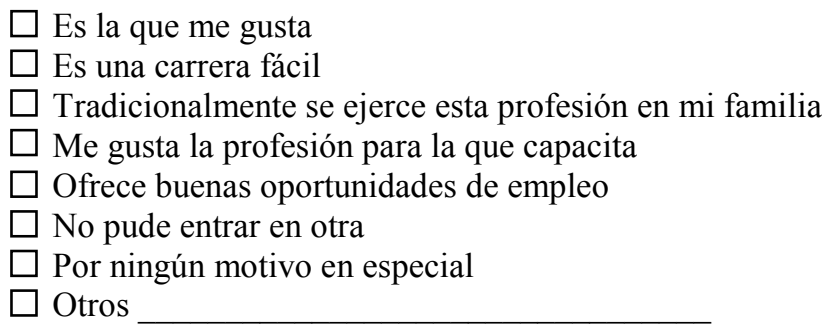

\section{B. OBJETIVOS DE LA EDUCACIÓN SUPERIOR}

Valora el grado de importancia de las siguientes afirmaciones (1: sin importancia; 2: poco importante; 3: importante; 4: bastante importante; 5: muy importante):

Son objetivos de la universidad:

5. Transmitir la cultura y los valores de la sociedad

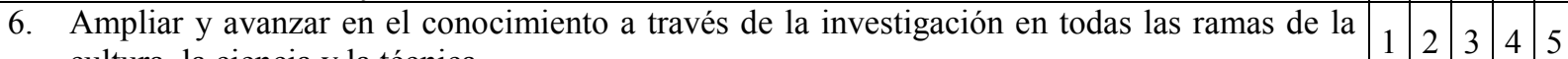
cultura, la ciencia y la técnica

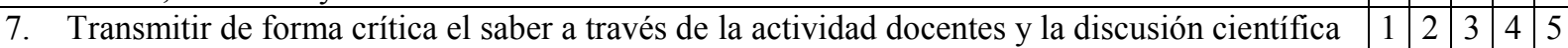

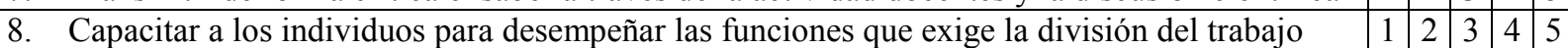

9. Contribuir a la mejora del sistema educativo

10. Estimular la actividad intelectual en todos los ámbitos

11. Asesorar científica, técnica y culturalmente a la sociedad de modo que se satisfagan sus necesidades

12. Cooperar con el desarrollo científico, técnico, cultural y social de todos los pueblos

13. Si consideras que la universidad ha de cumplir otros objetivos, señálalos

\section{DERECHOS Y DEBERES DE LOS ESTUDIANTES UNIVERSITARIOS}

Valora las siguientes afirmaciones (1: nunca; 2: en varias ocasiones, 3: en múltiples ocasiones):

\begin{tabular}{|l|l|l|l|}
\hline 14. Los estudiantes hemos consultado nuestros derechos en los estatutos de la universidad & 1 & 2 & 3 \\
\hline 15. Los estudiantes hemos consultado nuestros deberes en los estatutos de la universidad & 1 & 2 & 3 \\
\hline
\end{tabular}

A continuación, valora el grado de importancia de las siguientes afirmaciones (1: sin importancia; 2: poco importante; 3: importante; 4: bastante importante; 5: muy importante):

Son derechos de los estudiantes universitarios:

16. Recibir las enseñanzas teóricas y prácticas correspondientes a la titulación elegida

17. Elegir el profesor que imparta la docencia y evaluar su rendimiento

18. Participar activa y críticamente en las actividades docentes

19. Ser asistidos mediante un buen sistema de tutorías

20. Conocer con suficiente antelación la oferta docente y las fechas de examen 
21. Recibir una valoración objetiva de su rendimiento académico y conocer los criterios de valoración del mismo

22. Recibir gratuitamente los programas de cada asignatura

\begin{tabular}{l|l|l|l|l|}
1 & 2 & 3 & 4 & 5 \\
\hline
\end{tabular}

23. Recibir información sobre todo tipo de becas y ayudas al estudio y formar parte de las comisiones que las otorguen

24. Disponer de instalaciones adecuadas para el normal desarrollo de sus estudios y actividades culturales y deportivas

25. Participar en los órganos de gobierno y administración de la universidad

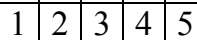

$\begin{array}{llllll}1 & 2 & 3 & 4 & 5\end{array}$

\begin{tabular}{llll|l}
1 & 2 & 3 & 4 & 5
\end{tabular}

26. Asociarse libremente

27. Recibir una adecuada información sobre sus derechos como estudiante, así como del funcionamiento general de la universidad

Son deberes de los estudiantes universitarios:

28. Respetar las normas y el patrimonio de la universidad

29. Realizar el trabajo propio de su condición de universitario

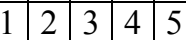

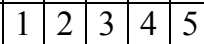

30. Asumir las responsabilidades de los puestos para los que sean elegidos

31. Cooperar con el resto de la comunidad universitaria en el buen funcionamiento de la universidad y en la mejora de sus servicios

32. Cooperar para la consecución de los objetivos de la institución

\section{MUCHAS GRACIAS POR TU COLABORACIÓN}

